\title{
Surface morphology of laser superheated $\mathrm{Pb}(100)$
}

\author{
Z. H. Zhang, Bo Lin, and H. E. Elsayed-Ali ${ }^{1}$ \\ Department of Electrical and Computer Engineering, \\ Old Dominion University, \\ Norfolk, VA 23529
}

The change in the surface vacancy density after heating of $\mathrm{Pb}(100)$ with $\sim 100 \mathrm{ps}$ laser pulses is investigated using reflection high-energy electron diffraction. The surface vacancy density remains unchanged when the surface is superheated without melting. However, when the laser fluence is high enough to cause surface melting, the surface vacancy density increases. This increase in vacancy density is attributed to fast diffusion of atoms in the liquid film formed on $\mathrm{Pb}(100)$ during laser melting.

PACS: 68.35.Bs, 68.35.Rh, 61.14. Hg, 42.65.Re

1 Corresponding author: Fax: (804)683-3220, e-mail: elsayed-ali@ece.ee.odu.edu 


\section{DISCLAIMER}

This report was prepared as an account of work sponsored by an agency of the United States Government. Neither the United States Government nor any agency thereof, nor any of their employees, make any warranty, express or implied, or assumes any legal liability or responsibility for the accuracy, completeness, or usefulness of any information, apparatus, product, or process disciosed, or represents that its use would not infringe privately owned rights. Reference herein to any specific commercial product, process, or service by trade name, trademark, manufacturer, or otherwise does not necessarily constitute or imply its endorsement, recommendation, or favoring by the United States Government or any agency thereof. The views and opinions of authors expressed herein do not necessarily state or reflect those of the United States Government or any agency thereof. 


\section{DISCLAIMER}

Portions of this document may be illegible in electronic image products. Images are produced from the best available original document. 
Recently, some close-packed surfaces were shown to be susceptible to large superheating [1-7]. With the use of short laser pulses, surface superheating has been observed in time-resolved reflection high-energy electron diffraction (RHEED) experiments [1-4]. Using 180 ps laser pulses, measured at full width at half maximum (FWHM), superheating of $\mathrm{Pb}(111)$ up to $\sim 120 \mathrm{~K}$ above the bulk melting point of $\mathrm{Pb}, \mathrm{T}_{\mathrm{m}}=600.7 \mathrm{~K}$, was observed [1]. Evidence of residual order on $\mathrm{Pb}(100)$ above $\mathrm{T}_{\mathrm{m}}$ was also observed [2]. In contrast, the open $\mathrm{Pb}(110)$ premelts for similar laser heating conditions [3]. In molecular dynamic (MD) simulations, superheating of fcc (111) surfaces was also reported [5-7]. In an $\mathrm{MD}$ simulation of laser superheating of a highly damaged $\mathrm{Cu}(111)$ surface, the large concentration of vacancies and adatoms was annealed through the filling of vacancies by surface atoms and the surface becomes atomically flat [6]. This annealing mechanism was attributed to the high vibrational amplitudes which atoms are forced into by the ultrafast superheating pulse. Most recently, we studied the surface morphology of $\mathrm{Pb}(111)$ after superheating with $\sim 100 \mathrm{ps}$ laser pulses [7]. Using quantitative reflection high-energy electron diffraction (RHEED) it was shown that superheating does not significantly change the surface average step terrace width or step edge roughness.

We report on a study of the surface morphology change after $\sim 100$ ps laser heating of $\mathrm{Pb}(100)$ using $\mathrm{RHEED}$. The $\mathrm{Pb}(100)$ surface undergoes vacancy-induced disordering, as observed using high-resolution low-energy electron diffraction (HRLEED) [9]. In the present study, we use similar experimental conditions for superheating of $\mathrm{Pb}(100)$ as previously used [2]. By analyzing the RHEED background intensity, the surface vacancy density change can be estimated $[8,10,11]$. The generation of a high vacancy density at the 
surface results in an increased RHEED background intensity [11]. Changes in the step terrace width are observed as changes in the RHEED intensity profile $[8,10] . \mathrm{Pb}(100)$, maintained at $533 \mathrm{~K}$ was further heated by a $\sim 100$ ps pulsed laser measured at FWHM. When the laser pulses cause surface superheating without melting, we observed that the RHEED background intensity does not significantly change when compared to that before laser heating. This indicates that the surface vacancy density does not change after superheating. However, when the laser fluence is high enough to cause surface melting after superheating, the background intensity increases, indicating that the vacancy density is increased on the surface after laser melting.

The experimental conditions were described elsewhere [7]. After sputtering and annealing the sample at $500 \mathrm{~K}$ for more than 24 hours, the Auger spectra did not show any detectable impurity. We obtained a sharp $1 \times 1$ RHEED pattern of the $\mathrm{Pb}(100)$ surface. $\mathrm{A}$ temperature uncertainty of $\pm 2 \mathrm{~K}$ near the $\mathrm{Pb}$ melting point and $\pm 1 \mathrm{~K}$ near the boiling point of water is estimated. Temperature stability within $\pm 0.1 \mathrm{~K}$ was attained using a temperature controller. A Nd:YAG (yttrium aluminum garnet) laser operating at a wavelength $\lambda=1.06$ $\mu \mathrm{m}$ and a pulse width of $\sim 100$ ps with $50 \mathrm{~Hz}$ repetition rate was used to heat the sample. For a laser pulse energy of $1 \mathrm{~mJ}$, the corresponding fluence is $(9.0 \pm 1.6) \times 10^{6} \mathrm{~W} / \mathrm{cm}^{2}$. The laser fluence was varied from 0.4 to $10.6 \times 10^{7} \mathrm{~W} / \mathrm{cm}^{2}$.

Figure 1(a) shows the (00) beam peak intensity as a function of temperature at the inphase and out-phase conditions. The angles of incidence of the electron beam along the 
[011] direction of the $\mathrm{Pb}$ crystal at the in-phase and out-of-phase conditions were $\sim 2.1^{\circ}$ and $3.0^{\circ}$, respectively. In this figure, the intensity at the in-phase condition decreases with temperature almost exponentially below $480 \mathrm{~K}$ and deviates gradually from the exponential Debye-Waller dependence above $480 \mathrm{~K}$. The Debye-Waller factor $2 \mathrm{M}=\omega \mathrm{T}$, below $480 \mathrm{~K}$ corresponds to a slope $\omega=0.01034 \mathrm{~K}^{-1}$. The angular profiles of the $(00)$ beam at the out-ofphase condition at room temperature parallel and perpendicular to the incident direction of the electron beam, the [011] direction of the $\mathrm{Pb}$ single crystal, correspond to Lorentzian profiles indicating that surface steps are monoatomic in height [8]. We did not observed any split peaks in the (00) beam as was observed for $\mathrm{Pb}(111)$, which indicates that the $\mathrm{Pb}(100)$ surface steps are not vicinal [8]. The FWHM of the RHEED profile parallel to the incident direction of the electron beam at this out-of-phase condition is $0.13 \AA^{-1}$, which is the same width as that experimentally obtained at the in-phase condition. This suggests that the step terrace width is wider than the $1200 \AA$ instrumental resolution $[10,12,13]$. With increasing temperature, we did not observe any change in the FWHM of the RHEED profile at the outof-phase condition indicating that the step terrace width does not change with temperature. Figure 1(b) shows that the background intensity changes with temperature at both the inphase and out-of-phase conditions. We observed significant changes in the background intensity with temperature above $480 \mathrm{~K}$ at the out-of-phase condition. In this figure, $\mathrm{I}_{\text {peak }}$ is the peak intensity and $I_{\text {back }}$ is the background intensity. The background intensity is normalized to the peak intensity by calculating the ratio $R=I_{\text {back }}\left(I_{\text {peak }}-I_{\text {ack }}\right)$. This normalized background, $R$, is obtained at $0.20 \AA^{-1}$ which is about $11 \%$ of the first Brillouin zone. The increase in the measured background intensity above $480 \mathrm{~K}$ at the out-of-phase 
condition reveals that the surface develops a high density of vacancies in agreement with the HRLEED observations [9].

We investigated the change in the RHEED background intensity after laser heating. The sample was kept at $533 \mathrm{~K}$ using a hot stage while the laser pulses were used to transiently heat the surface. Figure 2 shows the change in the ratio $R=I_{\text {back }} /\left(I_{\text {peak }}-I_{\text {back }}\right)$ as a function of peak laser fluence for heating with 1000 laser pulses at each fluence, where $I_{\text {back }}$ is the RHEED background intensity while $I_{\text {peak }}$ is the $(00)$ peak intensity. The ratio $R$ does not change below a peak laser fluence of $6.5 \times 10^{7} \mathrm{~W} / \mathrm{cm}^{2}$. Above that fluence, the background significantly increases. In addition, we did not observe any change in the FWHM of the (00) beam profile parallel and perpendicular to the [011] direction of the $\mathrm{Pb}$ crystal indicating that the step density does not change after laser heating.

The temperature rise on the $\mathrm{Pb}(100)$ surface due to the laser heating pulse is calculated by a one-dimensional heat diffusion model [14]. The error bar in the fluence is mainly due to the estimated nonuniformity in the heating laser beam profile, which was measured to be $18 \%$ across the surface. The laser peak fluence was varied from 0.4 to 10.6 $\times 10^{7} \mathrm{~W} / \mathrm{cm}^{2}$. A peak temperature rise of $22 \pm 4 \mathrm{~K}$ for the $1.0 \times 10^{7} \mathrm{~W} / \mathrm{cm}^{2}$ laser peak fluence was calculated using a heat diffusion model. The bulk melting point, $T_{m}$, is $600.7 \mathrm{~K}$ and the previously measured maximum superheating temperature $T_{\text {super }}$ is $\sim 635 \mathrm{~K}$. Above $T_{\text {super }} \sim$ $635 \mathrm{~K}$, the laser fluence is high enough to cause the surface to melt. This temperature corresponds to a peak laser fluence of $\sim 4.8 \times 10^{7} \mathrm{~W} / \mathrm{cm}^{2}$. From Fig. 2, we conclude that laser 
superheating of $\mathrm{Pb}(100)$ does not cause any noticeable change in the RHEED background intensity. When the surface is heated beyond the maximum superheating temperature, the surface melts and a sharp increase in the RHEED background intensity is observed.

Based on kinematics diffraction, for a two-dimensional surface containing only vacancies, the background intensity increases with the vacancy density without broadening the RHEED profile $[8,9,11]$. The ratio $R=I_{\text {back }} /\left(I_{\text {peak }}-I_{\text {back }}\right)$ can be determined by [9] $R \propto$ $\left[(2 n-1)^{-2}-1\right](1+2 M)$ for the out of phase condition, where $2 M=\omega T$ is the Debye-Waller factor, $\omega=0.01034 \mathrm{~K}^{-1}$ is the measured slope in our experiment, and $\mathrm{n}$ is the surface vacancy density. Therefore, the vacancy density is given by $n \propto 1 / 2(R /(1+2 M)+1)^{2}+1 / 2$. The vacancy density change is $\Delta n=n / n_{0}-1$, where $n_{0}$ is the initial vacancy density before heating. From Fig. 1(b), we calculate $\sim 20 \%$ increase in vacancy density at $480 \mathrm{~K}$, from that at $323 \mathrm{~K}$. At $530 \mathrm{~K}, \Delta \mathrm{n}$ increases by $\sim 300 \%$, indicating a higher generation of vacancy density in our case than that previously observed in HRLEED experiments in which the measured increase in vacancy density was $(20 \pm 5) \%$ at $530 \mathrm{~K}$ from its value at room temperature [9]. The observed larger increase in the vacancy density in our case maybe related to the different initial surface vacancy density at room temperature due to the different surface sputter treatment conditions in the two experiments. Both experiments, however, show that the change in the vacancy density increases exponentially with temperature and that large concentrations of vacancies are created above $510 \mathrm{~K}$. From Fig. 2, we obtain the vacancy density change as a function of peak laser fluence. The vacancy density is almost constant below $6.5 \times 10^{7} \mathrm{~W} / \mathrm{cm}^{2}$ and increases up to about $20 \%$ above $10.0 \times 10^{7} \mathrm{~W} / \mathrm{cm}^{2}$ due to surface melting. 
The results show that laser superheating of $\mathrm{Pb}(100)$ does not anneal surface vacancies. We also note that superheating of $\mathrm{Pb}(111)$ did not result in any reduction in surface step density [7]. In an $\mathrm{MD}$ simulation of ultrafast laser heating of $\mathrm{Cu}(111)$, large concentrations of vacancies and adatoms was shown to anneal through a non-diffusional cooperative mechanism in which the adatoms settle in the top layer, while the cooperative movement results in the filling of vacancies [6]. The experimentally observed stability of the surface vacancy density maybe due to the relatively low rate of surface diffusion of atoms on the solid layer $\mathrm{Pb}(100)$ surface. Surface diffusion of atoms was measured for $\mathrm{Pb}(110)$ and was estimated from a molecular dynamic simulations for $\mathrm{Au}(100), \mathrm{Au}(111)$, and $\mathrm{Au}(110)$ $[15,16]$. The one-dimensional diffusion coefficient below the melting point for $\mathrm{Au}(100)$ is estimated to be $\sim 1.0 \times 10^{-6} \mathrm{~cm}^{2} / \mathrm{s}$. In our experiment, we used $\sim 100 \mathrm{ps}$ laser pulses, which raises the surface temperature above $T_{m}$ for $100-150 \mathrm{ps}$. Within such a time scale, the atom diffusion length is $\sim 1-2 \AA$, which is less than the $4.95 \AA$ lattice constant of $\mathrm{Pb}(100)$. Thus, surface adatoms do not diffuse enough to fill existing vacancies. On the other hand, the onedimensional diffusion constant above the melting point for $\mathrm{Au}(100)$ is $\sim 2.5 \times 10^{-5} \mathrm{~cm}^{2} / \mathrm{s}$ resulting a diffusion length in a time scale of 100 ps much larger than the lattice constant of $\mathrm{Pb}(100)$. Such a large diffusion rate of adatoms on the surface causes the filling of vacancies. In the thin liquid film during surface melting, vacancies are also generated. If the rate of vacancy generation is higher than that of filling vacancies during laser melting, the vacancy 
density is increased. As a result, the surface vacancy density increases with the number of laser pulses and with the peak laser fluence.

Figure 3 shows the dependence of the ratio $R$ on the number of laser pulses used to heat the surface for a peak laser fluence of $7.34 \times 10^{7} \mathrm{~W} / \mathrm{cm}^{2}$, sufficient to melt the surface. The RHEED background intensity clearly increases up to 1500 pulses. The calculated result from Fig. 3 shows that vacancy density is increased from its starting point by $\sim 20 \%$ after 1000 laser pulses. The vacancy density is seen to slightly decrease as the number of heating laser pulses is increased beyond $\sim 1500$ pulses. A possible reason is that the generation of vacancies due to melting tends to saturate, thus, the rate of filling vacancies due to large adatom diffusion becomes higher than generating vacancies. Therefore, the vacancy density is slightly decreased in contrast to that below 1500 pulses. In the present experiment, we did not observe any change in the surface step density as observed in our previous work on vicinal $\mathrm{Pb}(111)$ where we observed that the surface step density is largely decreased after surface melting. Molecular dynamics simulations of fcc $(100)$ shows a weak disorder only in the first atomic layer and the surface does not undergo faceting [16]. The lack of faceting for $f c c(100)$ surfaces appears to be related to the high temperature for disordering of the two uppermost layers of the flat (100) surface $[16,17]$. In medium-energy ion scattering experiments, the disordered layer thickness on $\mathrm{Pb}(100)$ was observed to saturate at 1.3 monolayers, measured up to $\mathrm{T}_{\mathrm{m}}-0.05 \mathrm{~K}$ [18]. A periodic density modulation in the disordered layer in a direction parallel to the disordered layer interface is thought to make the disordered layer stable against further melting [19]. 
In conclusion, after laser superheating we observed that the RHEED background intensity does not significantly change compared to that before laser heating, thus, surface vacancy density does not change after superheating. When the laser fluence is high enough to cause the surface melting, we observed an increase in the background intensity, indicating an increase in the surface vacancy density. This increase in vacancies maybe attributed to the larger diffusion of atoms in the liquid film on $\mathrm{Pb}(100)$ during laser melting.

This work was supported by the US Department of Energy under the grants No. DEFG05-93ER45504 and DE-FG02-97ER45625. 


\section{Reference}

1. J. W. Herman and H. E. Elsayed-Ali, Phys. Rev. Lett. 69, 1228 (1992).

2. J. W. Herman, H. E. Elsayed-Ali, and E. A. Murphy, Phys. Rev. Lett. 71, 400 (1993).

3. J. W. Herman and H. E. Elsayed-Ali, Phys. Rev. Lett. 68, 2952 (1992).

4. E. A. Murphy, H. E. Elsayed-Ali, and J. W. Herman, Phys. Rev. B. 48, 4921 (1993).

5. F. D. Di Tolla, F. Ercolessi and E. Tosatti, Phys. Rev. Lett. 74, 3201 (1995).

6. H. Häkkinen and Uzi Landman, Phys. Rev. Lett. 71, 1023 (1993).

7. Z. H. Zhang, Bo Lin, X. L. Zeng, and H. E. Elsayed-Ali, Phys. Rev. Lett., submitted.

8. M. G. Lagally, D. E. Savage and M. C. Tringides, in Reflection High-Energy Electron Diffraction and Reflection Imaging of Surfaces, edited by K. Larson and P. J. Dobson, NATO ASI Series B, Phys. 188, Plenum, 139 (1988).

9. H.-N. Yang, K. Fang, G.-C. Wang, and T.-M. Lu, Phys. Rev. B 44, 1306 (1991).

10. C. S. Lent and P. I. Cohen, Surf. Sci. 139, 121 (1984).

11. J. M. Pimbley and T. M. Lu, Surf. Sci. 139, 360 (1984).

12. J. M. Van Hove and P. I. Cohen, J. Vac. Sci. Technol. A1, 609 (1983).

13. T. M. Lu and M. G. Lagally, Surf. Sci. 99, 695 (1980).

14. J. W. Herman and H. E. Elsayed-Ali, Phys. Rev. B 49, 4886 (1994).

15. J. W. M. Frenken, J. P. Toennies, and Ch. Wöll, Phys. Rev. Lett. 60, 1727 (1988). 
16. H. Häkkinen and M. Manninen, Phys. Rev. B 46, 1725 (1992).

17. G. Bilalbegović, F. Ercolessi, and E. Tosatti, Surf. Sci. 280, 335 (1993).

18. H. M. Van Pinxteren and J. W. M. Frenken, Surf. Sci. 275, 383 (1992).

19. A. A. Chernov and L. V. Mikheev, Phys. Rev. Lett. 60, 2488 (1988); A. A. Chernov and L. V. Mikheev, Physica 157A, 1042 (1989). 


\section{Figure Captions}

Fig. 1. (a) Peak intensity changes in the (00) beam as a function of temperature at the inphase and out-phase conditions. The angles of incidence of the electron beam along the [011] direction of the $\mathrm{Pb}$ crystal at the in-phase and out-of-phase conditions were $\sim 2.1^{\circ}$ and $\sim 3.0^{\circ}$, respectively. (b) The background intensity changes with temperature shows significant changes in the background intensity above $480 \mathrm{~K}$ at the out-of-phase condition.

Fig. 2. The change in the ratio $R=I_{\text {back }} /\left(I_{\text {peak }}-I_{\text {back }}\right)$ as a function of peak laser fluence for heating with 1000 laser pulses at each fluence. $I_{\text {back }}$ is the RHEED background intensity while $I_{\text {peak }}$ is the $(00)$ peak intensity. The ratio $R$ does not change below a peak laser fluence of $6.5 \times 10^{7} \mathrm{~W} / \mathrm{cm}^{2}$. Above that fluence, the background significantly increases.

Fig. 3. The dependence of the ratio $R$ on the number of laser pulses used to heat the surface for a peak laser fluence of $7.34 \times 10^{7} \mathrm{~W} / \mathrm{cm}^{2}$, sufficient to melt the surface. The RHEED background intensity increases up to $\sim 1500$ pulses. 

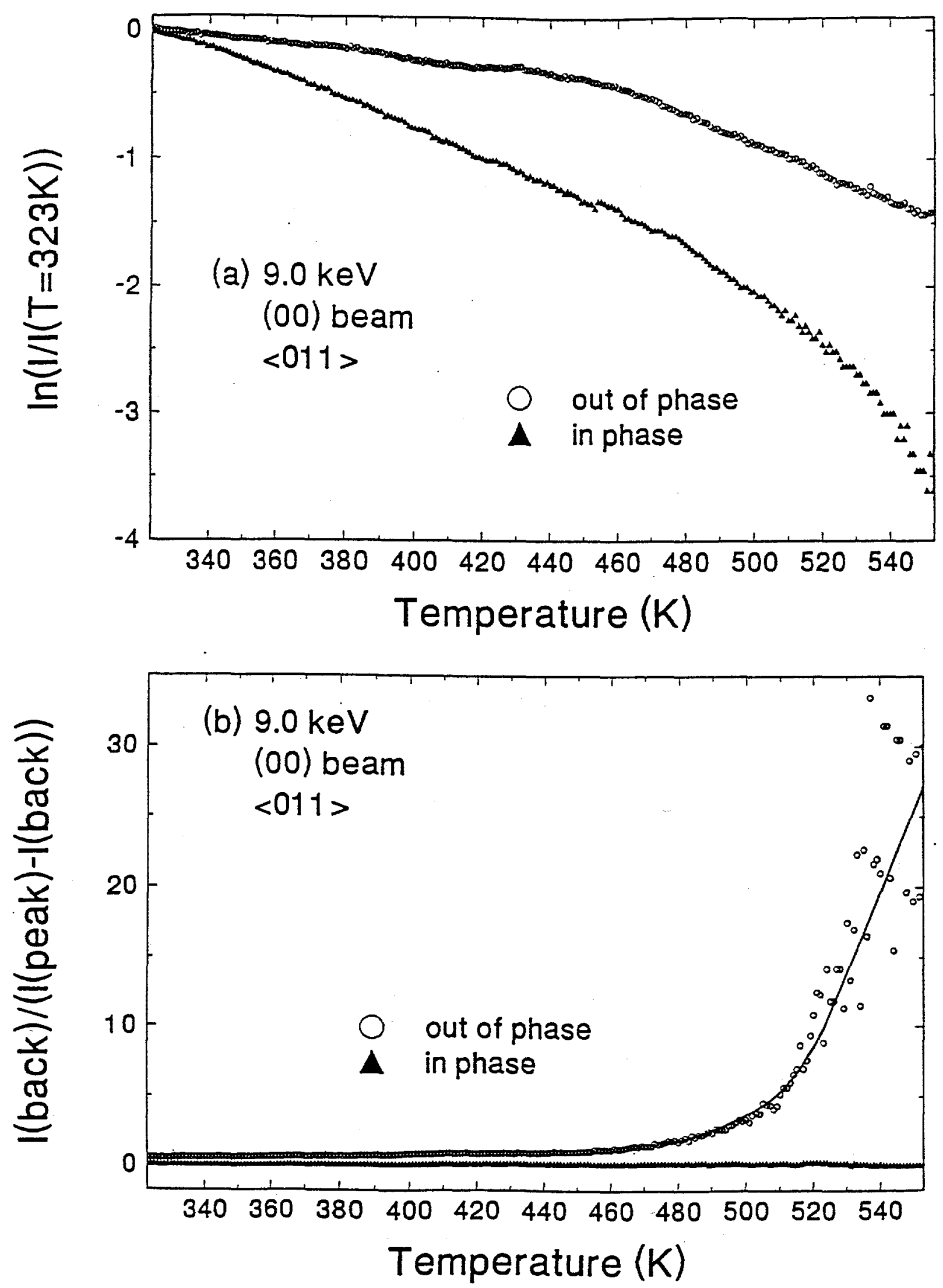

Fig. 1 
Fig. 2

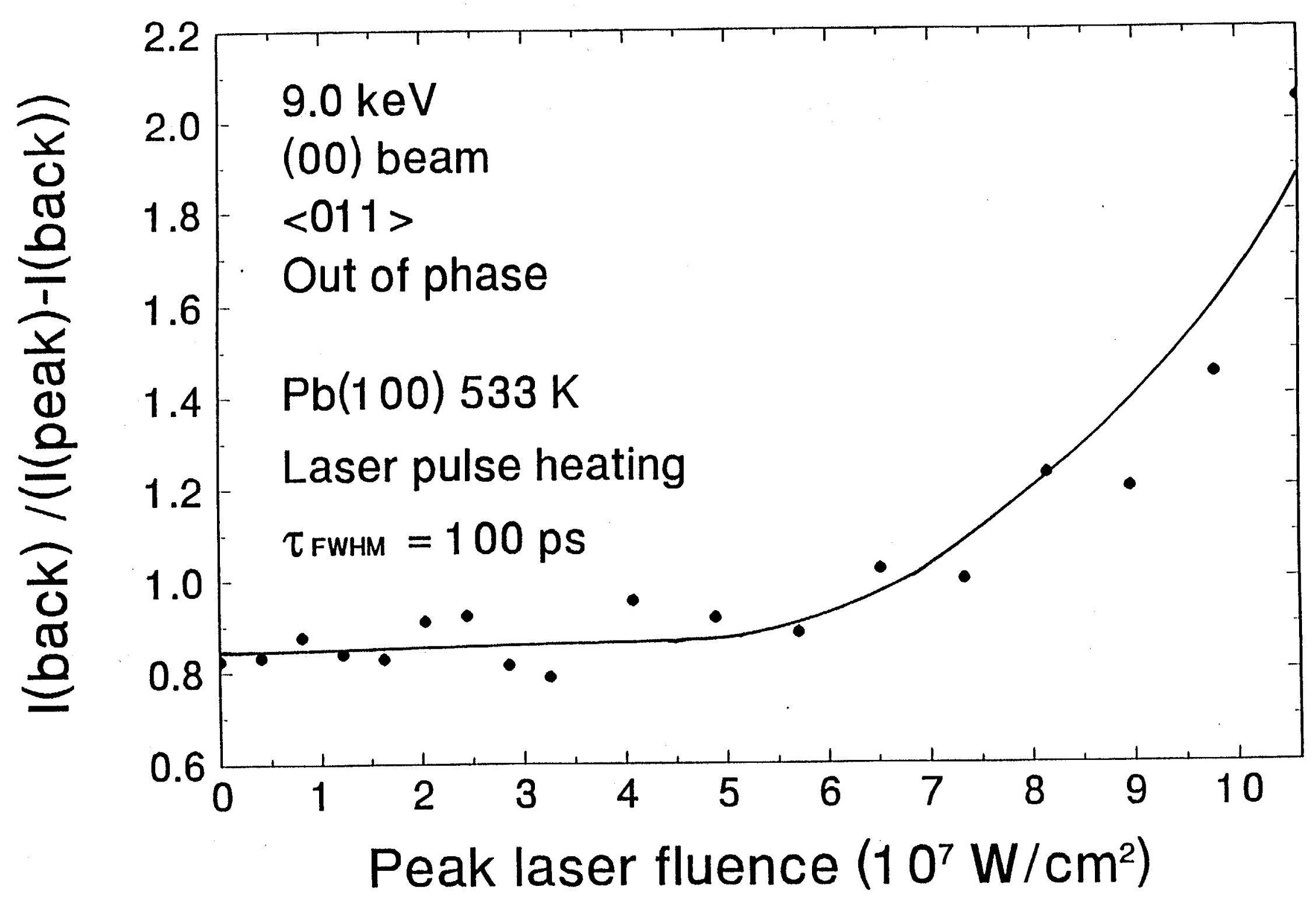


Fig. 3

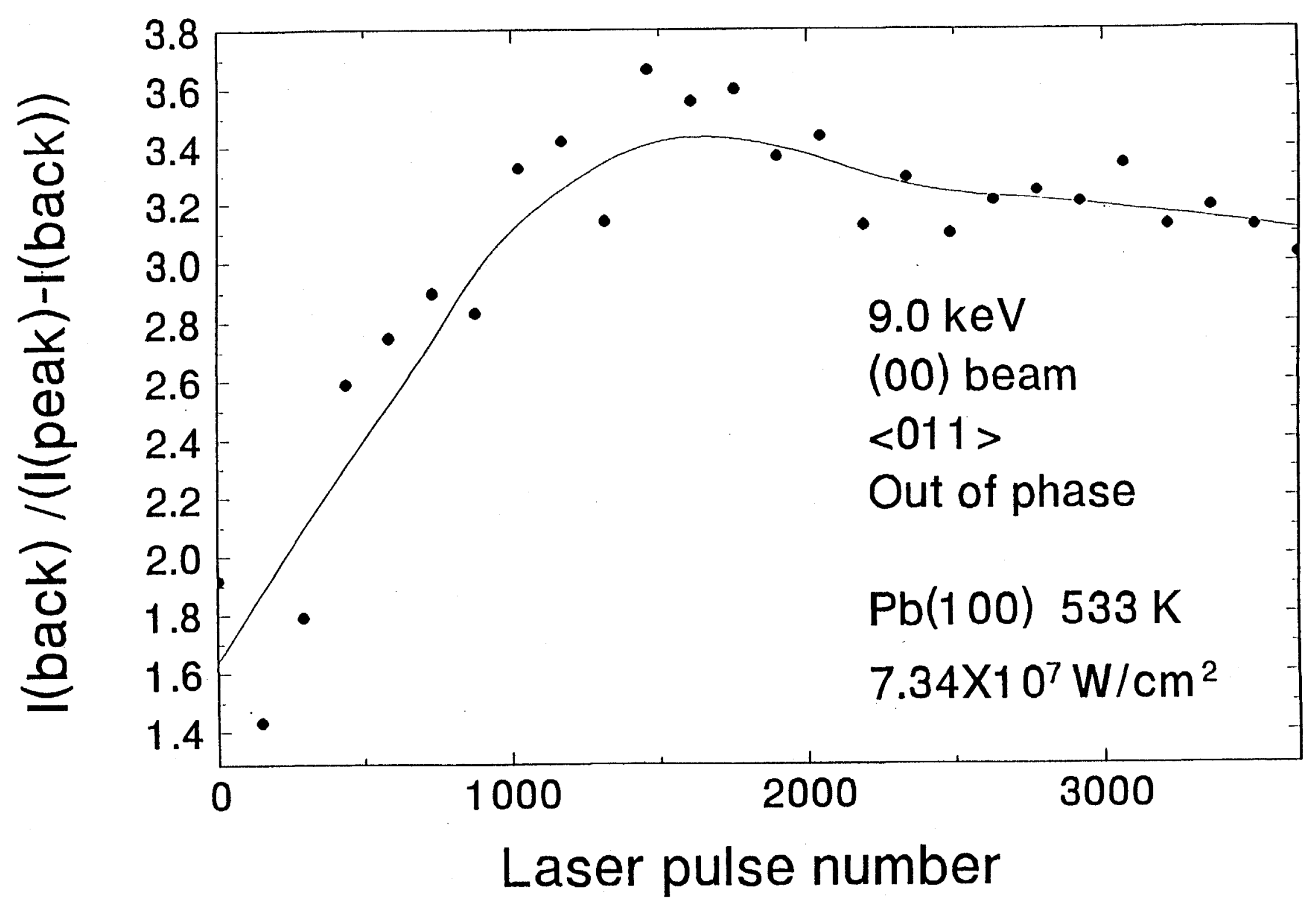

